\title{
Characterization of Calcium Phosphosilicate (Hydrate) Nanoparticles: Novel Organic-Inorganic Composite Nanomaterial for Drug and Diagnostic Delivery
}

\author{
James H. Adair ${ }^{1}$
}

${ }^{1}$ Materials Science \& Engineering, Penn State University, University Park, PA, USA

Calcium phosphosilicate nanoparticles (CPSNPs) were developed to deliver drugs and imaging agent in the treatment of human cancer. The material is an amorphous calcium phosphate [nominal composition $\mathrm{Ca}_{1.67}\left(\mathrm{PO}_{4}\right)_{(1-\mathrm{x})} \mathrm{SiO}_{3(\mathrm{x})}$ ], where $0<\mathrm{x}<0.1$. The CPSNPs are precipitated at $25^{\circ} \mathrm{C}$ within a reverse micelle system that permits the formation of nanoparticles ( $\sim 15$ to $35 \mathrm{~nm}$ diameter) intentionally composed of the amorphous calcium phosphate phase (Figure 1) [1]. One of the goals for the CPSNPs is to encapsulate small, and awkwardly shaped drugs including the chemotherapeutics, 5-fuoro-uracil and gemcitabine, and imaging agents such as indocyanine green and Rhodamine WT. Thus, the amorphous phase is preferred because this phase can accommodate the encapsulation of the drugs in the amorphous material matrix far better than the corresponding crystalline phases. During development, we discovered that small substitutions of meta-silicate ion $\left(\mathrm{SiO}_{3}{ }^{2-}\right)$ for the phosphate $\left(\mathrm{PO}_{4}{ }^{3-}\right)$ yield an amorphous calcium phosphate that is more stable than pure, amorphous calcium phosphate. During the development, obtaining TEM images to guide the process was found to be very difficult. The CPSNPs were difficult to obtain images with our standard analytical TEM approaches. Through much trial and error, it was established that electron beam damage was compromising the ability to obtain TEM images. Therefore, we moved from the materials side of our TEM facilities at Penn State over to the life sciences TEM facilities designed in part to image delicate biological samples.

As part of the biological approach to TEM imaging we also used the cryo stages and cryo sample holder to further reduce the damage in the delicate organic-CPSNPs during TEM observation. We also began to use various inorganic stains including phosphotungstic acid and osmium phosphate to enhance contrast and resolution. The inorganic contrast agents unexpectedly enabled us to obtain images of a detailed nanoscale structure within the amorphous CPSNPs. For the first time in the calcium phosphate system, we were able to observe the formation of precursor nanoparticles (1-3nm in diameter) that form the final 15 to $35 \mathrm{~nm}$ diameter nanoparticles through an agglomeration and secondary nucleation and growth process. This nanoparticle formation mechanism was discovered by Bogush and Zukowski in the 1980s based on TEM observations during the hydrolysis of silicon metalorganics to form monodispersed nanoscale amorphous silicon dioxide nanoparticles. To the best of our knowledge this formation mechanism had never been observed for the calcium phosphate system. The TEM observations of the finer ultrastructure during synthesis of the CPSNPs lead us to hypothesize that encapsulation of the various active agents was mediated by adsorption on the precursor nanoparticle clusters that subsequently agglomerate into the final particle. This ultimately lead to the striking revelation that phosphorylated forms of the drugs would encourage robust encapsulation through phosphate on the drug molecule binding to excess calcium sites on the precursor nanoparticles (Figure 2) $[1,2]$. This hypothesis was verified by an increase in encapsulation efficiency from $<1$ mole $\%$ for prior formulations to 60 to 95 mole\% for the phosphorylated drugs. Furthermore, the phosphorylated drugs did not have a maximum tolerated dose in vivo and were a thousand fold more effective in dose response than the non-phosphorylated drugs on multiple human cancer cells. Thus, the development of protocols to image the CPSNPs proved to be a key element in optimization of the encapsulation of the various active agent in the CPSNPs for efficient drug delivery and early detection of human disease. 
References:

[1] Welley S. Loc, et al, Nanomedicine: Nanotechnology, Biology and Medicine 13 (2017), p. 2313, DOI: 10.1016/j.nano.2017.06.017

[2] Gary A. Clawson, et al, Nucleic Acid Therapeutics 27 (2017), p. 23, DOI: 10.1089/nat.2016.0621

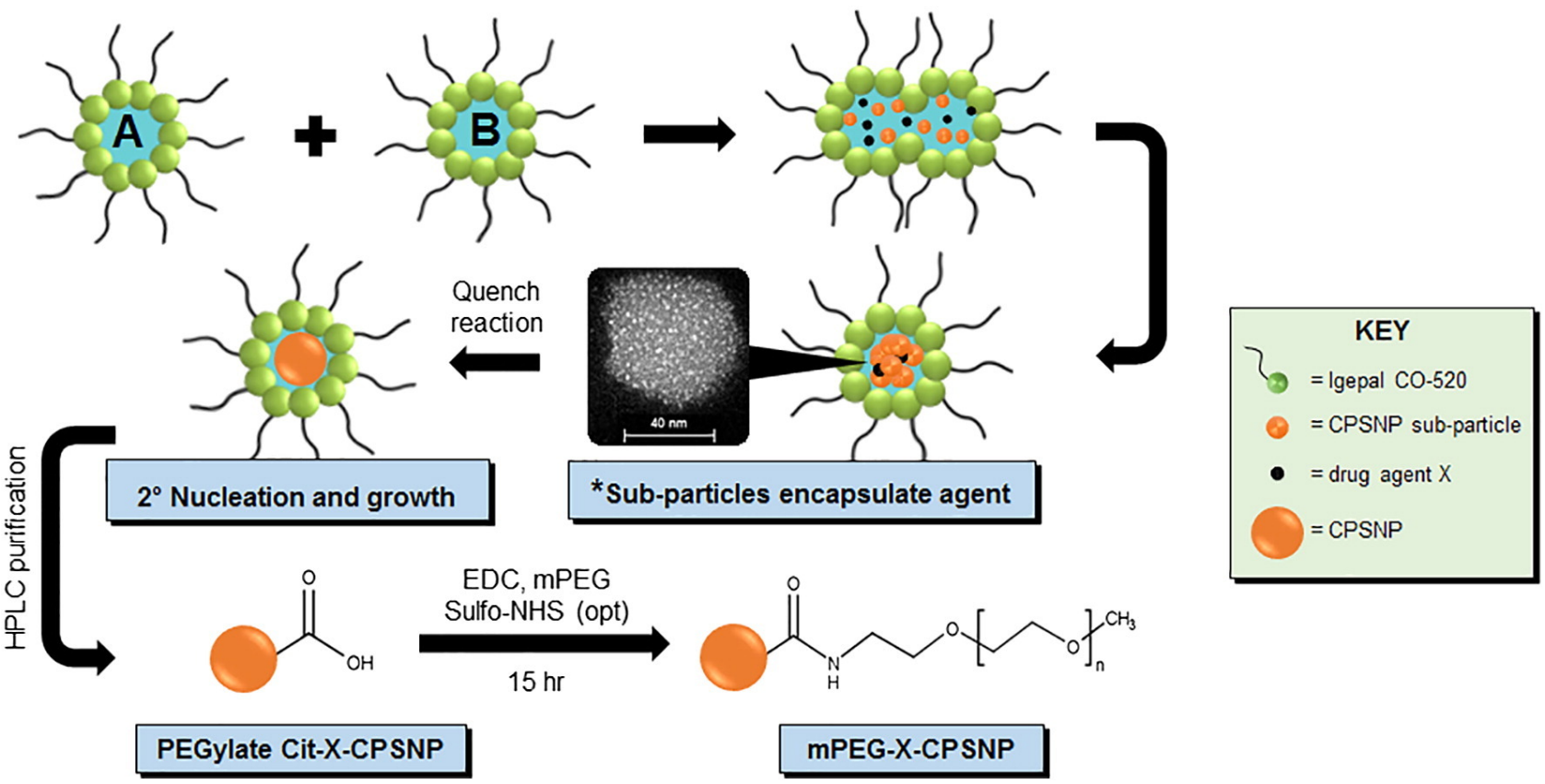

Figure 1. Synthetic scheme for CPSNPs using reverse micelles with Os-stained CPSNPs revealing the 1-3 nm diameter ultra microstructure of the 50nm diameter CPSNPs through which the phosphorylated compounds obtain high encapsulation efficiency [1].

Figure 2. CPSNPs for (Left) diagnostic determination of human pancreatic cancer in a murine model via

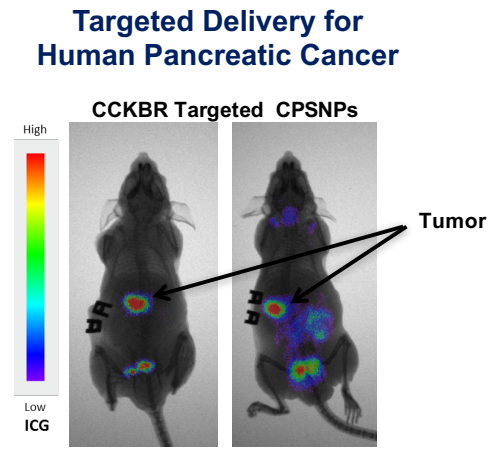

G. Clawson, et al. Nucleic Acid Therapeutics, 2017

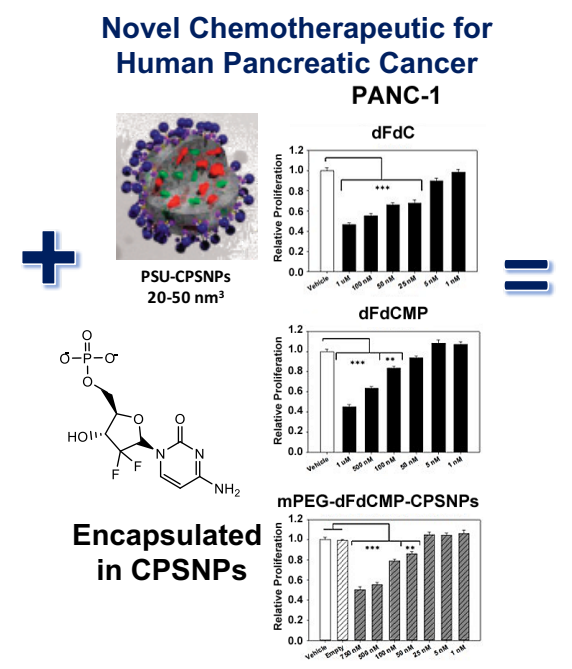
near infra-red imaging [2] and (Right) delivery of novel chemo-therapeutic via encapsulation with high encapsulation efficiency in CPSNPs for treatment of human pancreatic cancer [1]. The aptamer 1153 targeting was more effective than gastrin 16 targeting with both active targeting more effective than passive mPEG-CPSNPs. The phosphorylated, encapsulated drugs are almost a 1,000fold more effective for human pancreatic cancer than corresponding non-phosphorylated drugs. 